\title{
PASSIVE SUPERCONDUCTING SHIELDING: EXPERIMENTAL RESULTS AND COMPUTER MODELS
}

\author{
B. A. Warner ${ }^{1}$ and K. Kamiya ${ }^{1,2}$ \\ ${ }^{1}$ Code 552, Cryogenics Branch, Goddard Space Flight Center \\ Greenbelt, Maryland 20771, USA \\ 2 Tsukuba Magnet Laboratory, National Institute for Materials Science \\ Tsukuba, Ibaraki 305-0003, Japan
}

\begin{abstract}
Passive superconducting shielding for magnetic refrigerators has advantages over active shielding and passive ferromagnetic shielding in that it is lightweight and easy to construct. However, it is not as easy to model and does not fail gracefully. Failure of a passive superconducting shield may lead to persistent flux and persistent currents. Unfortunately, modeling software for superconducting materials is not as easily available as is software for simple coils or for ferromagnetic materials. This paper will discuss ways of using available software to model passive superconducting shielding.
\end{abstract}

\section{INTRODUCTION}

This work was suggested by the development of magnetic shielding for the Adiabatic Demagnetization Refrigerator (ADR) for the Submillimeter And Far Infrared Experiment (SAFIRE). The flux density at the detectors must be below $10^{-7}$ tesla during operation, while the central flux density of the magnet is 0.1 tesla. The shielding for SAFIRE used a combination of ferromagnetic, active, and passive superconducting shielding. As shown in FIGURE 1, the passive superconducting shielding consists of a flat ring of sheet niobium on the mounting flange of the magnet. The hole in the center of the sheet allows access to the magnet clear bore, where the salt pill of the ADR is placed. Measurements of shield performance show that the niobium sheet reduces the field seen by the sensors when the magnet current was ramped up, but also created a small residual field when the magnet current was reduced to zero [1,2]. 


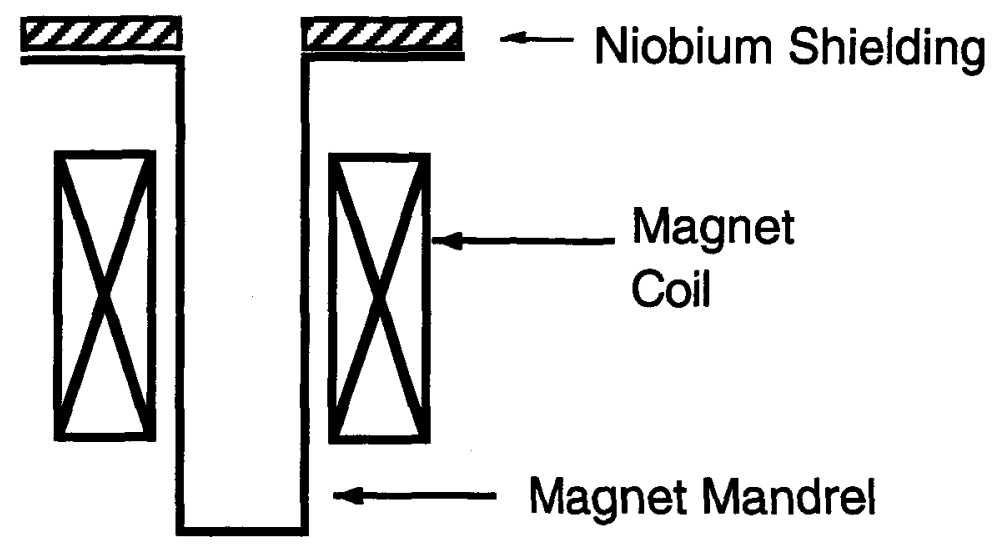

FIGURE 1. Simplified diagram showing position of the flat niobium ring shield on the SAFIRE magnet.

In this study, we compared the shielding effect of a flat ring of niobium sheet (as used on SAFIRE) with the shielding effect of a solid disk of niobium sheet. We found that each shield has advantages and disadvantages. We also modeled both types of shield using two types of available magnetic modeling software. The two programs were Poisson, by the Accelerator Code Group of the Los Alamos National Laboratory, and Magneto, by Integrated Engineering Software. Neither package was designed to model superconducting flux exclusion, but both modeled the field profile above the solid disk fairly well. However, the programs do not model superconducting critical fields. Therefore, passive superconducting shields must be tested especially thoroughly. Despite this drawback, passive superconducting shields are worth studying because they are light weight and easy to construct.

\section{EXPERIMENTAL WORK}

\section{Equipment and Procedure}

The magnet used for this study was a simple solenoid made by American Magnetics, Inc. The setup included no shielding other than the niobium sheet being tested, which was $0.5 \mathrm{~mm}$ thick, $99.9 \%$, by Goodfellow. The niobium sheet was placed $15.4 \mathrm{~cm}(61 / 16$ ") above the center of the magnet. As was the case with SAFIRE, the center of the disk or ring was on the axis of the coil. The niobium sheet was clamped between two plates of aluminum, $11 / 2 \mathrm{~mm}$ thick. . The outer diameter of the niobium pieces was $11.1 \mathrm{~cm}$. The diameter of the hole in the center of the flat ring was $4.1 \mathrm{~cm}$.

Four Hall effect sensors were mounted to the exposed surfaces of the aluminum plates, two on the top surface and two below, at distances of $2.8 \mathrm{~cm}$ and $4.995 \mathrm{~cm}$ from the coil axis. A movable Hall effect sensor also measured the vertical (that is, axial) component of the field on the coil axis. For the solid disk, the movable sensor measured only the field above the disk; for the flat ring, the sensor measured both above and below. 


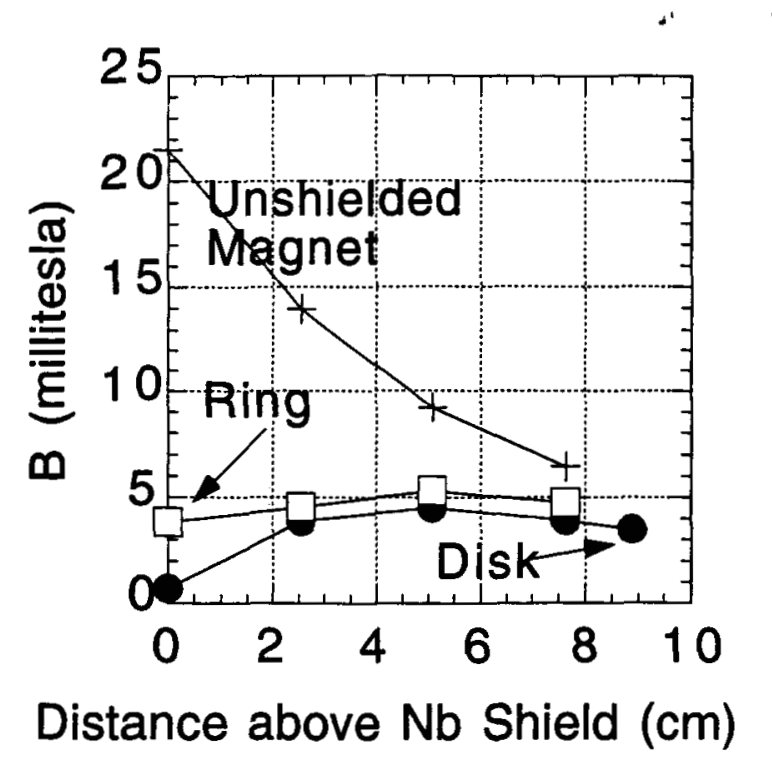

A: magnet current 4 amps.

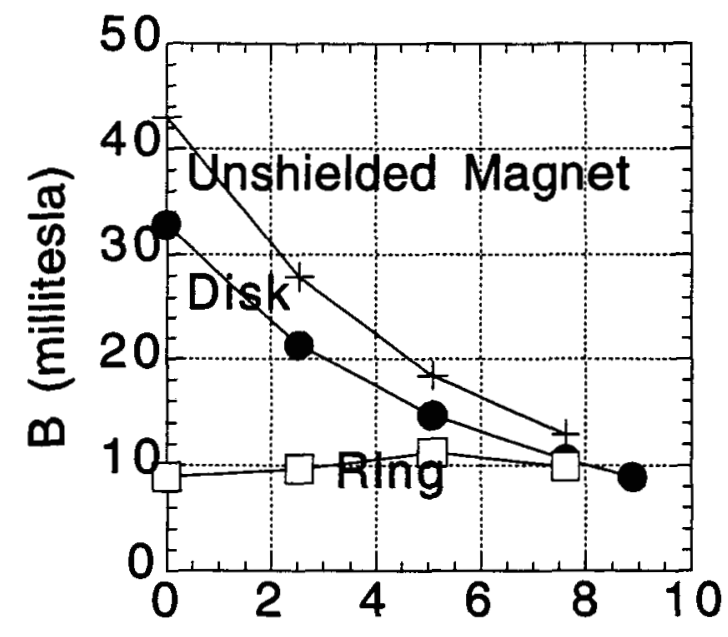

Distance above $\mathrm{Nb}$ Shield (cm)

B: magnet current 8 amps.

FIGURE 2. Field profiles above the niobium shield, comparing measurements made above the ring (plotted as hollow square) and the solid disk (solid bullets) with the profile calculated for the magnet alone. FIG $2 \mathrm{~A}$ is for a magnet current of 4 amps. For the disk, the measurements used are for positive magnet current. The field was higher when remeasured with negative current polarity. FIG 2B is for 8 amp case. Lines to guide the eye only; not curve fits

Our procedure was intended to let us measure both the shield performance at high applied field and the residual field when magnet current was ramped down to zero. We checked the calibration by reading at zero magnet current. Then we ramped up to 1 amp magnet current in the positive direction. After measuring the field, we ramped down to zero magnet current and measured the residual field. Next, we reversed the polarity of the magnet current and again ramped to $1 \mathrm{amp}$ and measured the field, after which we returned to zero magnet current and again measured the residual field. We repeated this process for progressively higher absolute values of current, reaching $8 \mathrm{amps}$ in the case of the flat ring and $10 \mathrm{amps}$ for the flat disk. (In the absence of shielding, on axis flux density at the location of the niobium shield was 5.4 millitesla per amp of magnet current, dropping to 4.6 millitesla per amp of magnet current at the outer edge of the niobium.)

All readings were taken with the liquid helium bath venting to atmosphere, thus at an approximate temperature of $4.2 \mathrm{~K}$. Measurements of $\mathrm{H}_{\mathrm{cl}}$ for niobium at $4.2 \mathrm{~K}$ give values around $9 \times 10^{4} \mathrm{~A} / \mathrm{m}[3,4]$ (corresponding to a free space $B$ value of 110 millitesla.)

\section{Measurements}

Both the solid disk and the flat ring showed easily measurable shielding effects. These effects were strongest for magnet currents at or below 4 amps (that is, for applied on-axis flux density at the niobium of 21 millitesla.) On axis, just above the niobium ring, the field was reduced by a factor five at a magnet current of 4 amps. Above the disk, the field was reduced by a factor of twenty when the test was run with 4 amps magnet current in the positive polarity. We repeated the measurement immediately with 4 amps current in the negative polarity. For this measurement, the field was reduced by a factor of only three, 


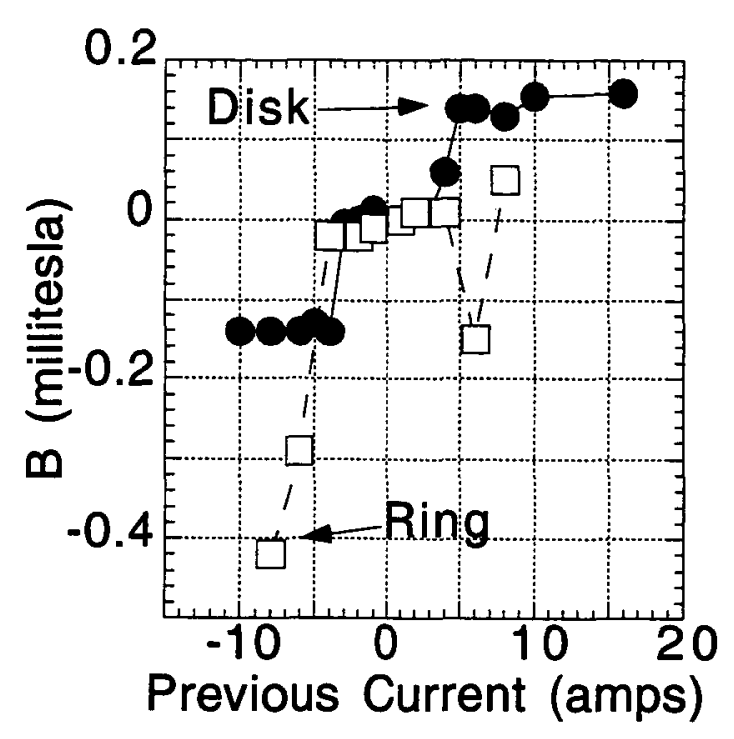

FIGURE 3. Residual field above shield with zero magnet current. Residual field is plotted against maximum magnet current of the current ramp just previous to the measurement. Lines to guide the eye only.

indicating that the field had begun to penetrate the disk..These measurements were taken with the movable Hall effect sensor. For the disk, the sensor was lowered till it touched the niobium. For the ring, the sensor was positioned in the center of the hole. FIGURE 2A shows the field profile just above the niobium shields, comparing the fields measured above the disk and ring with the field computed by Poisson for the unshielded case for a magnet current of 4 amps. For the disk, we use the profile measured for the positive current polarity, not the higher field measured with negativemagnet current polarity. In this figure, and in the other figures, lines are to guide the eye only; they are not curve fits. FIGURE 2B shows the same set of field profiles for a magnet current of 8 amps. As the figure shows, the solid disk has lost most of its shielding ability. The ring, however, continues to shield, with approximately the same ratio of applied field to shielded field as at 4 amps.

As mentioned above in the procedure section, we repeatedly ramped the magnet current from zero up to a maximum, then back to zero. We alternated positive and negative polarity. After each pair of ramps with the same absolute value, we increased the absolute value for the next pair. In FIGURE 3, we show the residual fields at zero magnet current. Each residual field reading is plotted vs. the maximum of the current ramp which just preceded it. The data in the graph are from the fixed sensor above the niobium plate at 2.8 $\mathrm{cm}$ from the axis. Data from the other sensors were similar, both in general trends and in the amount of scatter. The disk shows an increase in residual flux in the interval between 3 amps and 4 amps, with the size of the residual field leveling off for the rest of the studied currents. For the ring, the increase in residual field occurred for currents above 4 amps, and showed no signs of leveling off for the currents used. The on-axis residual field profile for the ring showed a local minimum at the height of the ring, that is, in the center of the ring's hole. This indicates that the residual field is not due only to a current flowing around the ring, as a ring current's axial field is highest in the center or the ring 


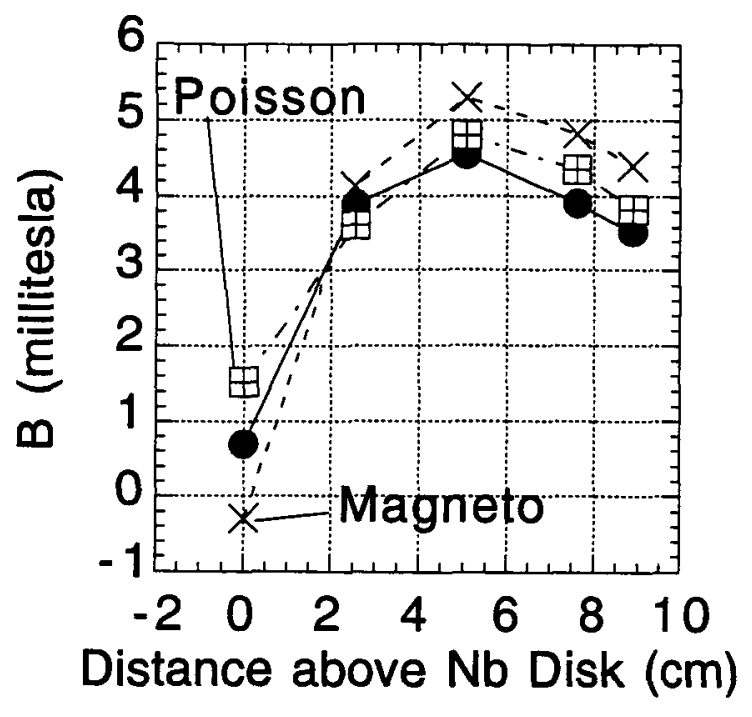

A: Magnet Current 4 Amps

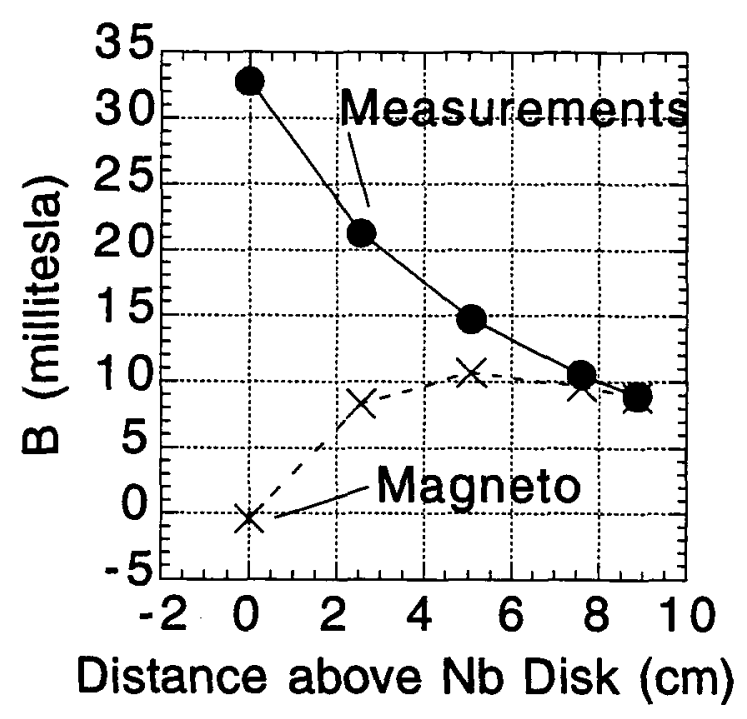

B: Magnet Current 8 Amps

FIGURE 4. Measured field profiles at magnet current of 4 amps (A) and 8 amps (B) above the niobium disk, compared with calculated profiles. The 4 amp profile is that measured with positive magnet current polarity, not the higher fields measured when the test was repeated with magnet current in the opposite polarity. (Above is away from the magnet, hence in the shielded area.) For 4 amps (A) calculations by both Magneto and Poisson are shown. For 8 amps (B) only calculations by Magneto are shown. The lines are to guide the eye only. They are not curve fits.

\section{COMPUTER MODELING}

Although there are many programs available for modeling fields produced by solenoids and by ferromagnetic material, we are not aware of any that model the effect of passive superconducting shields. We have therefore attempted to model these test shields with two packages, Poisson and Magneto. Both were successful for the disk at low magnet currents (4 amps and below), but both failed for higher currents and for the ring.

Modeling passive superconducting fields with these programs presents three difficulties. First, the programs are unable to model perfect diamagnets, that is materials with relative permeability of identically zero. In practice, this is the least of the difficulties, as both can model relative permeabilities of $<<1$. Second, the programs do not model the effect of a critical field. This is clearly a serious problem. Third, the programs have no way of setting the strength of field (if any) trapped inside a superconducting ring as the ring is cooled though its transition temperature. This is a serious problem for models of rings.

FIGURE 4A shows the measured field profile above the niobium disk for a magnet current of 4 amps, compared with the profiles modeled by Poisson and Magneto. Although the computer models are not perfect, they are good enough to be useful. This figure shows the profile as measured with the positive magnet current, not the higher fields found when the profile was remeasured with reverse magnet current polarity.

FIGURE 4B shows the field profile above the disk measured at a magnet current of 8 amps, compared with the profile calculated by Magneto. Clearly, field had begun to 
penetrate the disk. Equally clearly, Magneto did not model the penetration. (Poisson was likewise unable to do so.) Thus, it is impossible to rely completely on these magnet calculation programs as long as they have no way of modeling critical fields.

In some cases, however, it may be possible to judge when a superconductor will begin to transition. We have modeled diamagnetic ellipsoids using both Poisson and Magneto. Ellipsoids are useful tests of magnetic modeling techniques because the internal field of an ellipsoid placed in an initially uniform field is constant and can be calculated analytically [5]. Both Poisson and Magneto correctly modeled the internal fields of spheres and of other ellipsoids. In these successful models, the ratios of long axis to short axis were less than ten. However, neither program was able to correctly model an ellipsoid of approximately the same size and shape of the superconducting disk used in the tests. Such an ellipsoid has a ratio of long axis to short axis of approximately 100 .

Nevertheless, there may be a way to predict approximately when a superconducting disk will begin to allow field penetration. It is possible to model field penetration of a superconducting sphere by calculating $\mathrm{H}$ tangential at the equator of the sphere, then using the continuity of the tangential component of $\mathrm{H}$ across boundaries to find the field just inside the sphere [6]. Our Magneto models suggest that this may also be a useful technique for predicting field penetration of thin disks, whether or not the model field inside the disk is completely accurate. The field began penetrating the disk at a magnet current of about 4 amps. When we measured the field profile with a magnet current of 4 amps in the positive polarity, the field above the disk was reduced by a factor of twenty compared with the unshielded case. When we immediately repeated the measurements with a magnet current of 4 amps in the opposite polarity, the field was much increased, being approximately one third of the unshielded field, thus indicating that the field was beginning to penetrate. In our Magneto models, the tangential field immediately outside the midplane of the disk is $9 \times 10^{4} \mathrm{~A} / \mathrm{m}$ for a magnet current of 3 amps and $1.2 \times 10^{5} \mathrm{~A} / \mathrm{m}$ for a magnet current of 4 amps. Since $\mathrm{H}_{\mathrm{s} 1}$ is $9 \times 10^{4} \mathrm{~A} / \mathrm{m}$, this result is suggests that the field in fact began penetrating

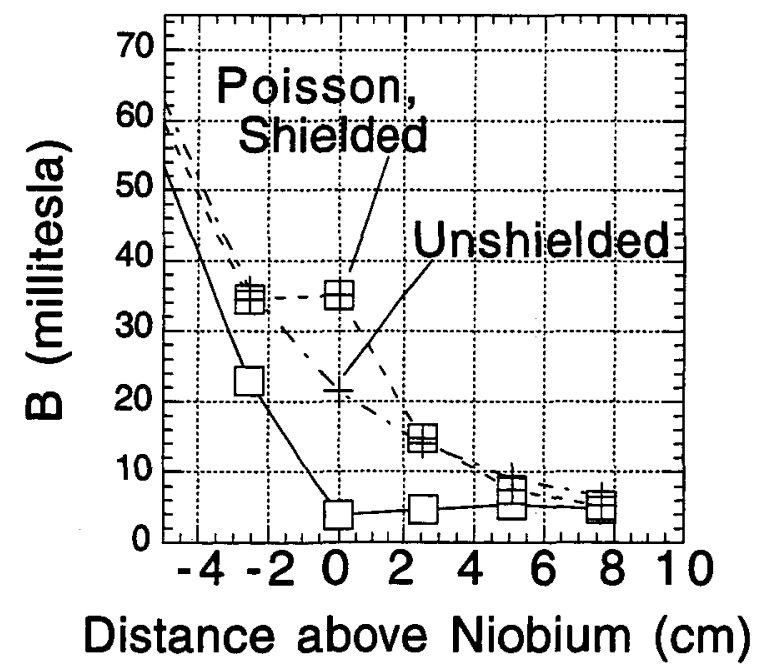

FIGURE 5. Field profile above and below the niobium ring shield. (Below is towards the magnet. Above is away from the magnet.) Actual measurements are plotted as hollow squares. Poisson calculations are indicated by the square with cross. Shown for comparison is the calculated field profile in the absence of any niobium shield. Lines are to guide the eye only. They are not curve fits. 
when the tangential field at the rim exceeded $\mathrm{H}_{\mathrm{cl}}$, . However, more work is needed to determine if this is a reliable way of predicting field penetration in practice.

FIGURE 5 shows the measured profile just above and just below the niobium ring for a magnet current of 4 amps, compared with calculations by Poisson. The profile calculated by Magneto is similar to that found by Poisson. The figure also includes the profile of the unshielded magnet as calculated by Poisson. Both programs calculated that the field inside the ring as larger than the unshielded field of the magnet alone. This might be the case if the niobium ring were cooled through its transition temperature in the presence of the applied field. In our study, however, the ring was cooled before the field was applied. It is possible to approximate the measured profile by including a current ring in the model to cancel the field in the hole. The value of this current is not simply the current one would calculate to produce the necessary opposing field on axis. The current must be adjusted to allow for the presence of the diamagnetic disk, which reduces the flux that it produces. Arriving at this model is quite cumbersome. It is doubtful that such a model would ever be useful.

\section{CONCLUSION}

Passive superconducting shielding as studied here is useful. The disk and ring shapes both have advantages and disadvantages. The decision of which to use will probably be based mostly on what is allowed by the configuration of the rest of the apparatus (as was the case with SAFIRE). When a solid disk is chosen, standard magnetic field programs should be useful for modeling. It may even be possible to estimate the field at which the

field will begin to penetrate. However, it is much more difficult to model the case of a ring.

\section{ACKNOWLEDGMENTS}

K. Kamiya was financially supported by a Research Fellowship of the Japan Society for the Promotion of Science.

\section{REFERENCES}

1. Kamiya, K, Warner, B. A.., and DiPirro, M. J. Cryogenics 41, pp. $401-405$ (2001).

2. Kamiya, K., Warner, B. A., DiPirro, M. J., and Numazawa, T. Physica B, 329-333, pp. 1627-1628 (2003).

3. Caterall, J. A., Williams, I., and Duke, J. F., British Journal of Applied Physics 15, pp. 1369-1375 (1964)

4. Goodman, B. B. and Kuhn, G., Le Journal de Physique 29, pp. 240-252 (1968).

5. Bozorth, R. M. and Chapin, D. M., Journal of Applied Physics 13, pp. 320-326 (1942).

6. Rose-Innes, A. C., and Rhoderick, E. H., Introduction to Superconductivity, Second Edition, Pergamon Press, New York, 1978, pp. 64-68. 